\title{
SEARCH FOR MAGNETIC STARS AT EARLY STAGES OF EVOLUTION
}

\section{YU. V. GLAGOLEVSKIJ}

Special Astrophysical Observatory, Nizhnij Arkhyz, 357147, Russia

Young stars, as a rule, are too faint for measurements of magnetic field either by photographic method with the use of Zeeman analizer, or photoelectrically from hydrogen lines. That is why it is necessary to look for indirect ways of magnetic field detection, for example, by measurement of polarization. $\mathrm{Ae} / \mathrm{Be}$ Herbig stars without a magnetic field are surrounded by a gaseous envelope in the form of a globe or a spheroid, flattened along the rotational axes (as dependent on stellar rotation velocity), and also by a gaseous-dust accretion disc in the plane of equator. There are powerful flows in gaseous envelopes of stars, connected with mass loss and accretion. If a star is a magnetic oblique rotator (as a magnetic star of the Main Sequence), then the gaseous envelope may acquire the shape of alongated ellipsoid with the major axes coincident with that of dipole (Dolginov et al., 1979). From the poles there arises a jet flow controlled by a magnetic field, as in He-r and He-w stars, having already reached the Main Sequence (Barker et al., 1982). Calculations show (Dolginov et al., 1979), that maximum polarization in the extended envelope $p \approx 4 \%$ arises when the ratio of ellipsoid axes is $a \approx 2.5 \mathrm{~b}$. The electric vector of the dominating oscillation of the light wave is perpendicular to the plane through the axis of symmetry of the ellipsoid and the line of sight. Naturally, the magnetosphere rotates together with the star, involving the gaseous envelope, resulting in the variation of the degree and direction of polarization. Additional polarization is created by the polar jets, where the direction of the dominating oscillations of the electric vector is perpendicular to the axis of the polar stream, and value of maximal polarization may reach $5 \%$ along the beam.

The gaseous envelope is formed mainly around the star and dust is chlefly contained in the disc. The scatter of light in the gaseous component occurs in the optical wavelength range, but in the dust one - in the infrared range. As a consequence of the fact that the electric vector in the former case is oscillating perpendicular to the axis of symmetry of the envelope, and in the latter its oscillations develop along the axis, the linear polarization changes by $\alpha=\left(90^{\circ}-\beta\right)$ from the optical to infrared spectral range. Here $\beta$ - is the angle between the magnetic field dipole axis and the axis of rotation. The data pre- 
sented show that in a case of a dipole field in an Ae/Be Herbig star, one may expect periodical variability of the linear polarization in the optical spectral region and constant polarization in the infrared region. It is possible to try to measure the inclination of the dipole axis.

Let us see what results of previous research can be used

for selection of samples in search for magnetic fields. Young stars of Ae/Be Herbig type become visible near the "birth line" found by Palla and Stahler (1990), when a star loses a large part of its surrounding envelope. It turns out that for rapidly evolving massive stars of He-r type this occurs when they have already got to the Main Sequence. That is why the sample of stars for investigation should not include early B-stars. We found that the intersection boundary of the "birth line" with the Zero age Main Sequence is likely to fall on Sp = B5 - B7, but not on B2, found by Palla and Stahler (1990). This assumption can be made on the basis of Fig. 1, constructed from the data of Table 6.1 from (Shevchenko, 1989). Spectral classes are on the horizontal axis, and on the vertical axis are the amplitudes of the photometric variability $\Delta \mathrm{m}$. The points are the average values $\Delta m$ in the narrow intervals of spectral types. It is well seen that at $\mathrm{Sp}=\mathrm{BO}-\mathrm{B} 7$ values $\Delta \mathrm{m}=0.4$, then $\Delta \mathrm{m}$ grow (first it was noted by Finkenceller and Mundt (1984)) and reach maximum at $\mathrm{Sp} \approx A 3$, then they drop to $\Delta \mathrm{m} \approx 1^{\mathrm{m}}$. The average values of amplitudes $\Delta \mathrm{m}$ for stars TTau equal $1^{\mathrm{m}}$ too, and it shows that the late Be Herbig stars and TTau stars are active approximately at the same degree. It is possible to make the following assumptions:

1) The nature of activity of TTau and Ae/Be Herbig stars is the same and there is no boundary near $M=1.5 M_{\odot}(S p \approx A 5-F 0)$,

where some investigators (on the basis of theoretical arguments) assume transition from convective to nonconvective atmospheres at early stages of evolution. Finkenceller and Mundt (1984) reported their opinion that, unlike the opinion of some theoreticians, young $\mathrm{Ae} / \mathrm{Be}$ Herbig stars pass the convective stage of evolution. The same opinion was expressed by Palla and Stahler (1990), and also by Kuan and Kuhi (1975) for explanation of activity and thestellar wind nature.

2) The majority of Be Herbig stars are in the limits of the Main Sequence band. Their activity has stopped and the photometric variability becomes small. The later stars ( $\mathrm{Sp} \approx \mathrm{B} 5$ - B7) are visible at large distances ( $\Delta t$ years) from the Zero age line (they get $r i d$ of the surrounding gaseous-dust envelope relatively earlier), and nonstationary processes in stars are in full swing.

One may suppose that in the first approximation, the stronger nonstationarity, the farther the star by time $\Delta t$ from the Zero age line; at the same time the amplitude $\Delta \mathrm{m}$ is larger the larger the luminosity $L$. In this case $\Delta \mathrm{m} \sim(\mathrm{L} \times \Delta t)$. In Fig. 1 is shown the dependence $(L \times \Delta t)+$ const upon $S p$. The values of $L$ and $\Delta t$ are estimated from the average dependence between the 
Zero age line and "birth line" (Palla and Stahler, 1990). The calculated dependence are shown in Fig. 1 for three predictions: when the "birth line" intersects the Zero age line at points Sp $=\mathrm{B} 2, \mathrm{~B} 5$ and $\mathrm{B} 7$. The best fit of calculated boundary and the experimental one is at $\mathrm{Sp}=\mathrm{B} 5$ - B7. As to the shape of the curve, it can be easily fit with the experimental one by selection of the "birth line" shape. But it has no sense because, first, there are very little data for confident determination of the maximum and the right part of the dependence in Fig. 1 and, second, the dependence $\Delta m \sim$ ( $L$ x $\Delta t$ ) maybe not so simple as we supposed. Magnetic stars at early stages of evolution are possible to find rather among Ae Herbig stars than among Be Herbig stars. It is doubtful that the separation boundary of convective and nonconvective atmospheres falls at $\mathrm{Sp}=\mathrm{B} 5-\mathrm{B} 7$.

\section{REFERENCES}

Barker,. P.K. , Brown, D.N., Bolton, C. T., Landstreet, J. D. : 1982, in Advances inU1traviolet Astronomy, NACA, p. 589.

Dolginov, A.Z., Gnedin, Yu. N., Silantiev, N.A.: 1979, in The Diffusion and Polarisationof the Radiation in Cosmic Medium. Moskwa, Nauka.

Finkenceller, U., Mundt,R.: 1984, Astr. Ap. Suppl., 55, 109.

Kuan, P. , Kuhi, L. V. : 1975, Ap.J., 199, 148.

Palla,F., Stahler,S.V.: 1990, Ap. J. Lett., 360, L47.

Shevchenko, V.S., 1989, in Ae/Be Herbig Stars. Tashkent.

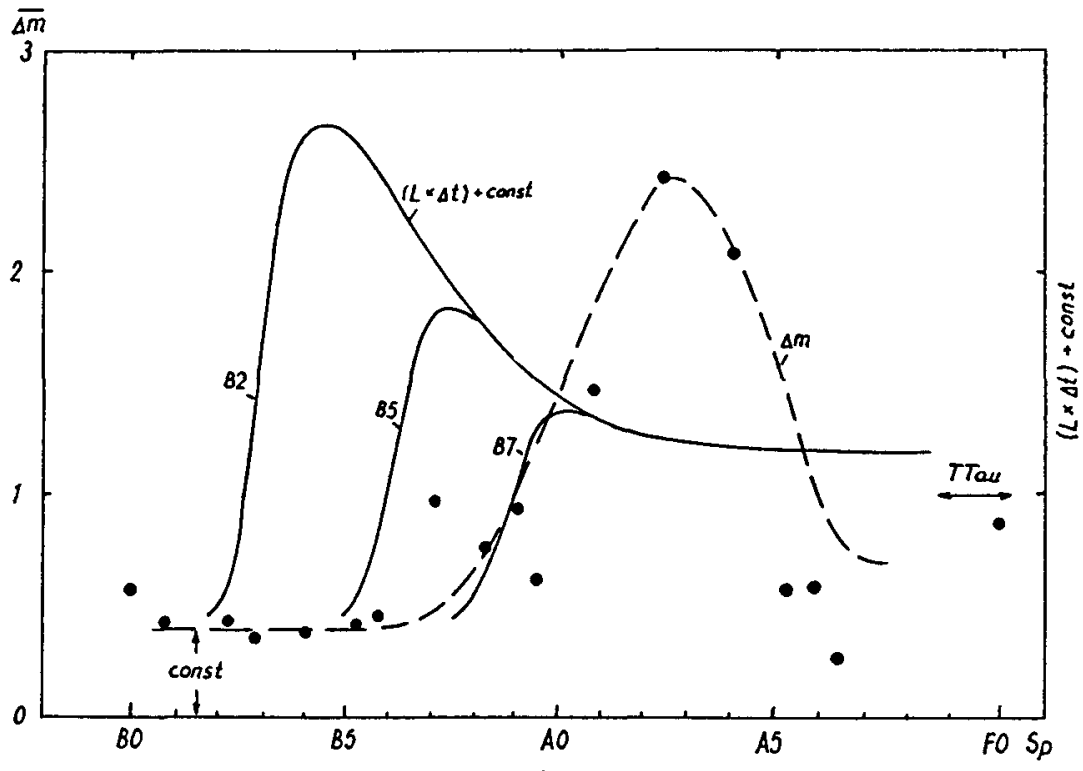

Fig. 1 